\title{
Characterization of Darai Limestone Composition and Porosity Using Data-Constrained Modeling and Comparison with Xenon K-Edge Subtraction Imaging
}

\author{
Sheridan C. Mayo, ${ }^{1, *}$ Sam Y.S. Yang, ${ }^{1}$ Marina Pervukhina, ${ }^{2}$ Michael B. Clennell, ${ }^{2}$ Lionel Esteban, ${ }^{2}$ \\ Sarah C. Irvine, ${ }^{3,4}$ Karen K. Siu, ${ }^{5,6}$ Anton S. Maksimenko, ${ }^{5}$ and Andrew M. Tulloh ${ }^{1}$ \\ ${ }^{1}$ CSIRO Manufacturing Flagship, Private Bag 10, Clayton, VIC 3169, Australia \\ ${ }^{2}$ CSIRO Energy Flagship, P.O. Box 1130, Bentley, WA 6102, Australia \\ ${ }^{3}$ Swiss Light Source, Paul Scherrer Institut, 5232 Villigen PSI, Switzerland \\ ${ }^{4}$ Department of Mechanical \& Aerospace Engineering, Monash University, Wellington Road, Clayton, VIC 3800, Australia \\ ${ }^{5}$ Australian Synchrotron, 800 Blackburn Rd, Clayton, VIC 3168, Australia \\ ${ }^{6}$ School of Physics, Monash University, Wellington Rd, Clayton VIC 3800, Australia
}

\begin{abstract}
Data-constrained modeling is a method that enables three-dimensional distribution of mineral phases and porosity in a sample to be modeled based on micro-computed tomography scans acquired at different X-ray energies. Here we describe an alternative method for measuring porosity, synchrotron K-edge subtraction using xenon gas as a contrast agent. Results from both methods applied to the same Darai limestone sample are compared. Reasonable agreement between the two methods and with other porosity measurements is obtained. The possibility of a combination of data-constrained modeling and K-edge subtraction methods for more accurate sample characterization is discussed.
\end{abstract}

Key words: data-constrained modeling, micro-CT, K-edge subtraction, porosity, synchrotron

\section{INTRODUCTION}

$\mathrm{X}$-ray micro-computed tomography $(\mathrm{CT})$ is a widely used method for characterizing reservoir rocks giving threedimensional (3D) structural information on density, morphology, and porosity at a resolution of a micron at best (Van Geet et al., 2000; Arns et al., 2005; Pinczewski et al., 2005; Cnudde \& Boone, 2013). Conventional micro-CT has some limitations in that different phases can only be identified where they differ significantly in X-ray attenuation, and features below the resolution limit may result in difficulty in identifying mineral phases due to the partial volume effect. For instance submicron porosity within a region will result in a lower X-ray attenuation in the X-ray micro-CT scan and can lead to ambiguity between nonporous mineral phases of lower $\mathrm{X}$-ray attenuation and nanoporous regions of higher X-ray attenuation phases, since individual voxels within the microCT scanned volume may contain more than one phase (including porosity). Insight into the submicron porosity can of course be gained by higher resolution techniques such as focused ion beam scanning electron microscope; (Bera et al., 2011,2012 ) however, this is a destructive technique and can only image a very small volume. One approach to resolving the ambiguities in micro-CT imaging of rocks containing submicron porosity is to use two or more micro-CT scans at different energies combined with data-constrained modeling (DCM) (Yang et al., 2010a). This enables us to model, in each voxel of the reconstructed 3D volume, the volume fractions of

Received December 16, 2014; accepted April 16, 2015

*Corresponding author. Sherry.Mayo@csiro.au the materials constituting the object/sample. DCM uses mathematical optimization to generate such a $3 \mathrm{D}$ compositional map that is consistent with the micro-CT data sets. This approach has already been used in a range of applications in petroleum geology (Mayo et al., 2012; Trinchi et al., 2012; Wang et al., 2013a, 2013b, 2013c; Yang et al., 2013a, 2013b; Chen et al., 2014).

DCM makes use of the different ways in which X-ray attenuation of different mineral phases change with energy to distinguish between them. The best quality data for DCM is acquired at synchrotron sources where specific monochromatic X-ray energies can be selected for CT scan, using knowledge about the sample to select those that give the best chance of distinguishing the different phases. In addition to identifying different phases, the level of porosity can also be modeled using DCM to resolve the ambiguity between regions of porosity below the resolution of the micro-CT scan and nonporous phases with lower X-ray attenuation.

An alternative method for characterizing the porosity of reservoir rocks is micro-CT using a contrast agent that can penetrate the pore network. This makes porosity visible by the degree of saturation of the contrast agent throughout the structure. It was demonstrated that quantitative results may be obtained by comparing scans with and without the contrast agent present (Withjack \& Akervoll, 1988). This method has since been widely applied to a range of sample types (Ketcham \& Iturrino, 2005; Ghous et al., 2007; Boone et al., 2014). Contrast agents often used in lab-based CT for this purpose include potassium or sodium iodide solutions (Agbogun et al., 2013; Andrew et al., 2014) and in some cases 
mercury (Klobes et al., 1997; Fusi \& Martinez-Martinez, 2013).

In the laboratory setting this type of analysis is complicated by beam hardening, although corrections can be made for this. More accurate results can be obtained by a synchrotron where monochromatic X-rays can be used and the X-ray energy selected and optimized. Furthermore, if the contrast agent has a suitable X-ray absorption edge, K-edge subtraction imaging can be used to isolate the contrast agent signal from that of the rest of the structure giving a more accurate analysis of porosity. Unlike the rest of the sample, the contrast agent has a large change in $\mathrm{X}$-ray attenuation at the absorption edge. By subtracting images acquired at X-ray energies immediately above and below the contrast-agents absorption edge, the contrast-agent absorption image can be isolated from that of the rest of the sample. This has previously been used in biomedical applications such as K-edge subtraction angiography, using iodine-based contrast agents (Umetani et al., 1991).

In what follows the DCM approach based on two micro-CT data sets at different energies is used to model the distribution of calcite, dolomite, and porosity (voids) in a sample of Darai limestone. The same sample is also scanned using a xenon contrast agent for K-edge subtraction analysis of porosity in Darai limestone. The resulting porosity measurements are used to validate modeling of the porosity of the sample as determined by DCM.

\section{Dual-Energy Micro-CT and K-Edge Subtraction Micro-CT OF Darai LIMESTONE}

The Darai limestone is a fossil rich dolomitized limestone from Papua new Guinea with many nanoporous regions. The sample was prepared as a mini-core $3.8 \mathrm{~mm}$ in dameter and $\sim 6-\mathrm{mm}$ long. Porosity was estimated from the difference in wet and dry mass to be $16.4(4) \%$. Figure 1 shows the fossil structures and dolomitic regions of a thin section of Darai limestone.

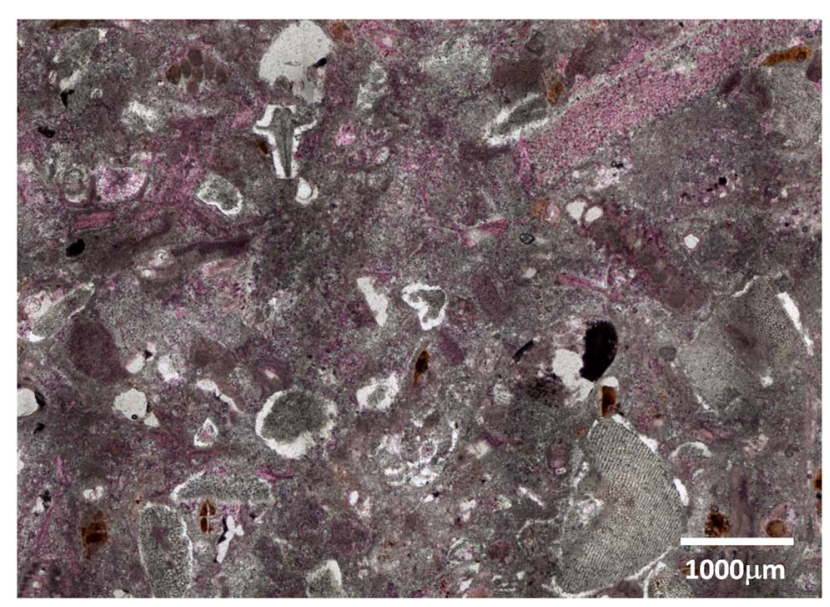

Figure 1. Optical thin section of Darai limestone, carbonate is pink and dolomite is uncolored.
Dual-energy micro-CT of the sample for DCM was performed at the TOMCAT micro-CT beamline at the Swiss Light Source. The X-ray energies used for the scans were 28 and $38 \mathrm{keV}$ and were selected using the multilayer monochromator that admits X-rays in a narrow energy band around the selected energy. The sample was mounted on a rotation stage and an Optique Peter detector with a PCO2000 sensor was used to acquire images of the sample with a sample-detector spacing of $21 \mathrm{~mm}$. The detector was set to an effective pixel size of $1.85 \mu \mathrm{m}$ giving a horizontal field of view of just under $4 \mathrm{~mm}$, sufficient to just accommodate the sample. The beam size limited the vertical field of view to around 3-mm vertically so only part of the 6-mm long sample was imaged; 1,500 images with an exposure time of $500 \mathrm{~ms}$ were acquired as the sample was rotated between 0 and $180^{\circ}$. Additional images were acquired without the sample present (flat-field images) and also with the X-ray beam off (dark-field images) to enable image correction for illumination and CCD artefacts.

The sample-to-detector spacing enabled the images acquired to benefit from phase contrast, an edge-enhancement effect arising from Fresnel diffraction (Snigirev et al., 1995; Nugent et al., 1996; Wilkins et al., 1996). The X-tract software (Gureyev et al., 2011) was used to perform image correction, phase retrieval, and tomographic reconstruction on the images. Phase retrieval using the TIE-HOM method (Paganin et al., 2002) was applied to the phase-contrast data to improve signal-to-noise and produce images in a suitable format for conventional tomographic reconstruction by filtered back-projection. The tomographic reconstruction output is a stack of images defining the $3 \mathrm{D}$ volume of the sample, with each reconstructed image representing cross sections of the sample perpendicular to the tomographic rotation axis. A sample tomographic section is shown in Figure 2.

The choice of X-ray beam energies $(28$ and $38 \mathrm{keV})$ is important in minimizing the sensitivity to noise of the DCM analysis (see Fig. 3). Qualitatively, there should be maximal difference, or contrast between corresponding CT slice images at the two energies. Several factors must be taken into consideration: the sample size, the absorption coefficients of the constituent materials, and the detector sensitivity and image exposure time. These parameters limit the choice of energies to $\sim 25-40 \mathrm{keV}$. In the absence of absorption edges for either calcite or dolomite in this range, the best choice is made where the difference in the ratio of absorption coefficients for calcite and dolomite is maximized between the two energies.

In a separate experiment a micro-CT scan was performed at the Australian Synchrotron of the same Darai sample using xenon K-edge subtraction with the intention of measuring porosity by looking at the quantity of xenon penetrating the structure. Although both data sets had a restricted vertical field of view $(2 \mathrm{~mm})$ that did not capture the whole length of the sample, it was realized that fortuitously there was an overlap in the 3D data produced during this experiment and the data sets acquired previously at Swiss Light Source. This provided an opportunity for validation of the porosity analysis produced by DCM. 


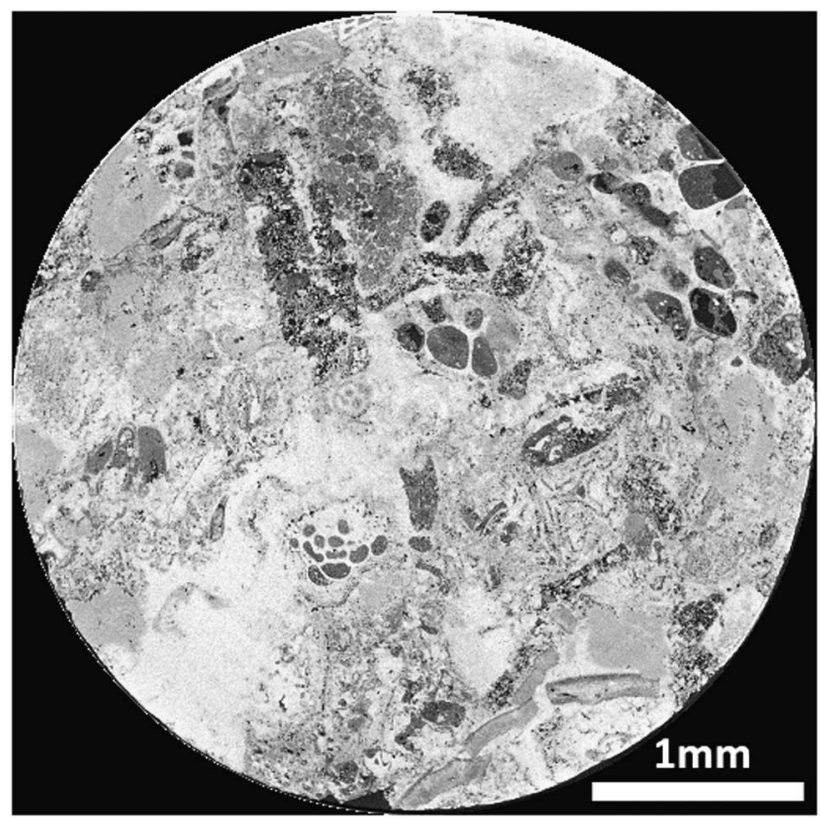

Figure 2. Tomographic cross section from $28 \mathrm{keV}$ scan of Darai sample acquired at Swiss Light Source. Note that the structure is dominated by fossil features.

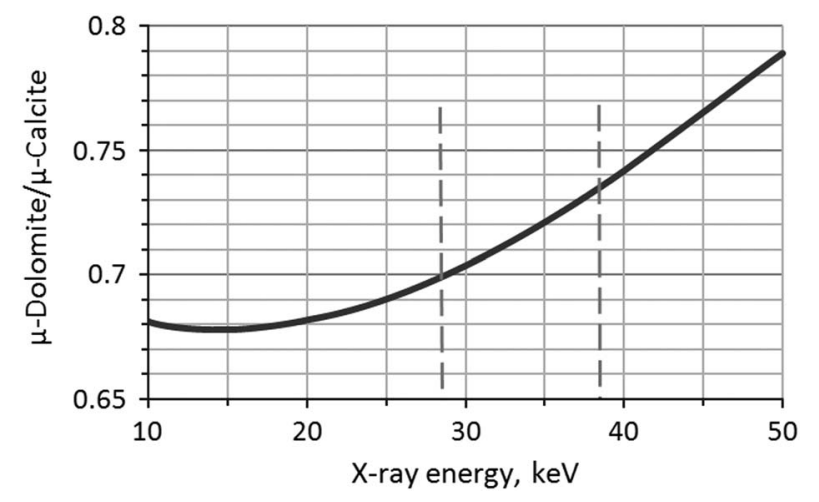

Figure 3. Ratio of total linear attenuation coefficient for dolomite and calcite. The chosen X-ray beam energies of 28 and $38 \mathrm{keV}$ provide a near-maximal difference in the ratio within the utilized energy range for the sample.

In this case the experimental set-up on the Imaging and Medical Beamline at the Australian Synchrotron was similar to that used to the Swiss Light Source. The main difference being that the sample was in a sealed container pressurized with $\sim 8$ atm of xenon gas (see Fig. 4), which could reasonably be expected to penetrate into even the smallest parts of the connected pore network of the sample. The gas pressure was selected to provide sufficient contrast to detect porosity in the sample while not attenuating the X-ray beam too strongly over the diameter of the tube.

An Optique Peter camera with a PCO Edge CMOS detector was used for collecting the tomographic images with 1,800 views acquired over a $0-180^{\circ}$ rotation. Background and dark-field images were again acquired for correcting

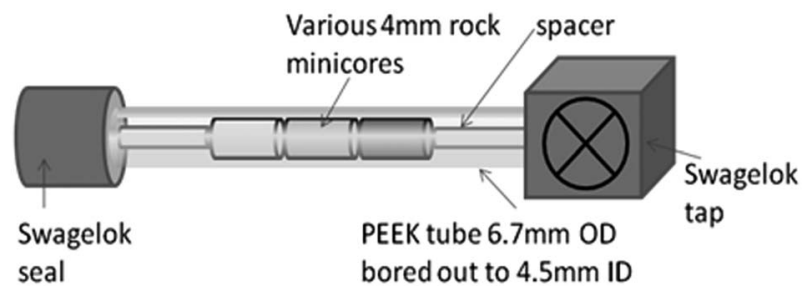

Figure 4. Sealed contained used for pressurized xenon experiments. $\mathrm{ID}=$ inner diameter, $\mathrm{OD}=$ outer diameter.

CCD and illumination artefacts. The sample-detector distance in this case was $5 \mathrm{~cm}$ and the pixel size $3.25 \mu \mathrm{m}$, thus the pixel size was larger and the resolution was observed to be poorer than that in the previous data sets. The vertical field of view was $\sim 2 \mathrm{~mm}$. Data were acquired at energies of 34 and $35 \mathrm{keV}$, straddling the xenon absorption edge at $34.5 \mathrm{keV}$.

Data were processed for tomographic reconstruction in the same way as the Swiss light Source data sets using $\mathrm{X}$-TRACT for image correction, phase retrieval, and tomographic reconstruction. The true gas pressure was determined from the measured X-ray attenuation of the free gas phase at $35 \mathrm{keV}$ and was found to be 8.0(1) atm.

\section{Porosity Determination from K-Edge SUBTRACTION DATA}

The two K-edge subtraction data sets were aligned in 3D with a rigid body alignment using the Avizo software package. This enabled the data set at $34 \mathrm{keV}$ in which the xenon has low X-ray attenuation to be subtracted from that at $35 \mathrm{keV}$ in which the xenon has much higher attenuation (see Fig. 5). A small scaling factor was applied before subtraction to account for the small change in carbonate attenuation between the two energies. The subtraction process effectively removes the limestone signal leaving a $3 \mathrm{D}$ image of the xenon distribution. Even in regions where the porosity cannot be resolved directly, the porosity can be inferred by the ratio of xenon present relative to the regions of pure xenon gas surrounding the sample.

The subtraction data contains regions with no xenon and a region of free xenon gas. Using tools in Avizo these areas were segmented for analysis. The intensity value of the zero-level $\left(I_{0}\right)$ was determined from the mean intensity in the xenon-free regions. Likewise the intensity value for regions of $100 \%$ xenon was determined from the mean intensity in the gas region around the sample $\left(I_{\mathrm{Xe}}\right)$. Finally the average intensity within the envelope of the rock sample was determined $\left(I_{\mathrm{P}}\right)$ and the average porosity $(\mathrm{P})$ calculated as $\mathrm{P}=\left(I_{\mathrm{P}}-I_{0}\right) /\left(I_{\mathrm{Xe}}-I_{0}\right)$.

Standard deviation in the xenon content measured in this way in individual pixels is estimated to be $\sim 18 \%$; however, because we are taking the mean over millions of pixels, this contribution to the error in the mean porosity measurement is rather small. Larger errors come from imaging artefacts such as ring artefacts that affect some 

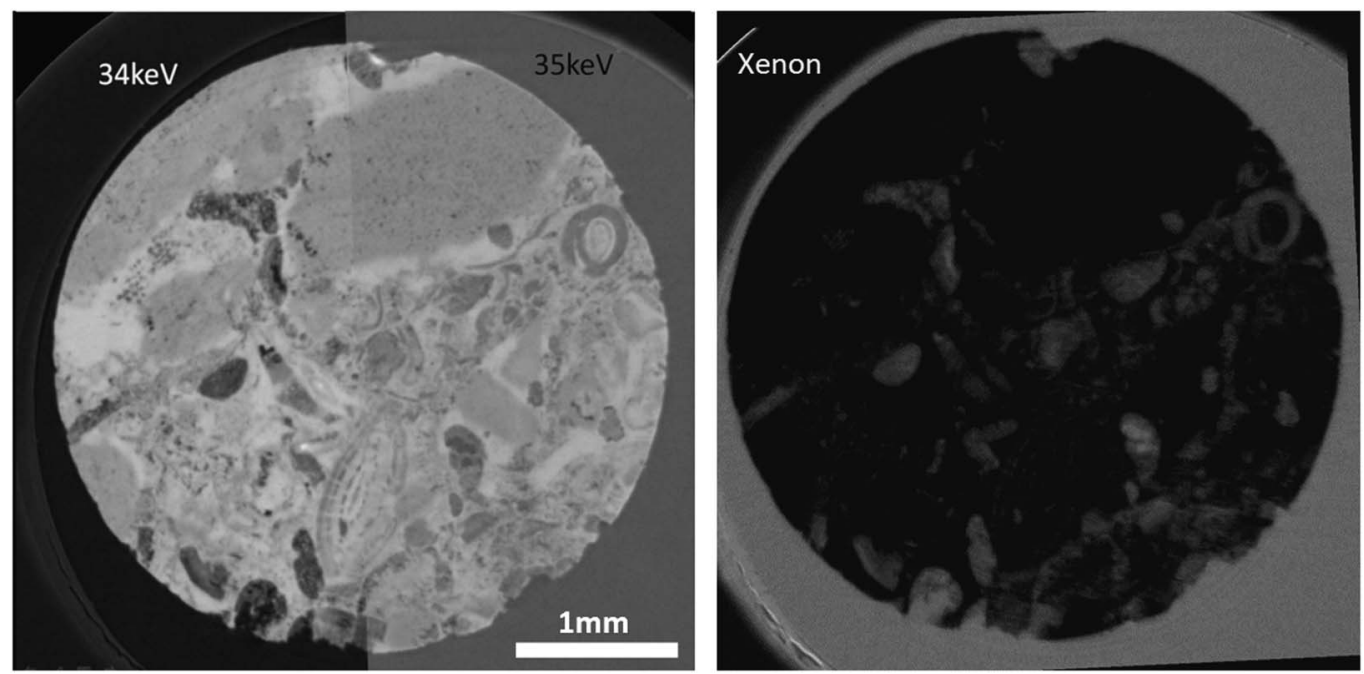

Figure 5. Left: split image showing the difference between a cross section below ( $34 \mathrm{keV})$ and above ( $35 \mathrm{keV})$ the xenon absorption edge. Right: subtraction image showing xenon distribution within the sample.

regions of the data, which is estimated to be of the order of $2 \%$. Average porosity in the overlap region between the K-edge subtraction data and the data acquired for DCM was found to be $19.5(4) \%$.

\section{DCM ANALYSIS}

The two data sets acquired at 28 and $38 \mathrm{keV}$ were first prepared for DCM analysis by 3D alignment and resampling of the data using Avizo to make sure the 3D data were perfectly registered with one another. These two data sets were then aligned with the K-edge subtraction data set to locate the region of overlap between the different data sets. This region of overlap was then used for subsequent DCM analysis.

The DCM was set up to model the volume fractions of three phases within the sample; calcite $\left(\mathrm{CaCO}_{3}\right)$, dolomite $\left(\mathrm{Mg}_{0.5} \mathrm{Ca}_{0.5} \mathrm{CO}_{3}\right)$, and void. The densities of the two mineral phases in their pure form are specified as 2.71 and $2.83 \mathrm{~g} / \mathrm{cm}^{3}$, respectively.

The DCM reconstructed distribution of pores and mineral phases has a size of $975 \times 975 \times 385$ voxels. Each voxel has a physical size of $1.85 \times 1.85 \times 1.85 \mu \mathrm{m}^{3}$. These 385 CT slices have been analyzed using the DCM software(Yang et al., 2014b). The analysis is done using a discrete leastsquare segmentation followed by a nonlinear optimization (Yang et al., 2010b, 2013b)

The nonlinear optimization is performed on a simplecubic grid with a cubic voxel (volumetric pixel) on each grid point. This is the same grid as the original CT data. For a voxel at $n(n=1,2, \ldots, N)$ where $N$ is the total number of voxels in the system, the model is to minimize the following objective function:

$$
T_{n}=\sum_{l=1}^{L}\left[\delta \mu_{n}^{(l)}\right]^{2}+E_{n}
$$

where $L=2$ is the number of X-ray CT data sets, and

$$
\delta \mu_{n}^{(l)}=\frac{1}{\mu_{\max }^{(l)}}\left|\sum_{m=0}^{M} \mu^{(m, l)} v_{n}^{(m)}-\hat{\mu}_{n}^{(l)}\right|
$$

where $\mu^{(m, l)}$ is the total linear absorption coefficient for material $m, \hat{\mu}_{n}^{(l)}$ the CT reconstructed linear absorption coefficient, and $\mu_{\max }^{(l)}=\max \left\{\mu^{(m, l)} ; m=0,1,2, \ldots, M\right\}$. The optimization is accomplished by adjusting the volume fraction variables $v_{n}^{(m)}(m=0,1,2, \ldots, M)$ for each material composition $m$, where $M=2$ is the total number of compositions in the system, subject to the constraints:

$$
\begin{aligned}
& \sum_{m=0}^{M} v_{n}^{(m)}=1 \quad \text { where } 0 \leq v_{n}^{(m)} \leq 1 \\
& \quad \text { for } m=0,1,2, \ldots, M .
\end{aligned}
$$

The dimensionless phenomenological interaction energy is expressed as:

$$
E_{n}=\sum_{k=0}^{K} \sum_{j=1}^{N^{(k)}} \sum_{m_{1}=0}^{M} \sum_{m_{2}=m_{1}}^{M}\left(v_{n}^{\left(m_{1}\right)} v_{n+n_{j}^{(k)}}^{\left(m_{2}\right)}\right)^{\frac{1}{2}} I_{k}^{\left(m_{1}, m_{2}\right)} .
$$

Where $I_{k}^{\left(m_{1}, m_{2}\right)}$ denotes the interfacial energy between neighboring voxel compositions $m_{1}$ and $m_{2}$ at distance $k$, where $k=0 \quad(k>0)$ denotes intra- (inter-) voxel. In the calculations, only up to the nearest-neighbor interactions have been used. That is, $K=1$. The number of neighboring voxels with neighboring range $k$ is denoted by $N^{(k)}\left(N^{(0)}=1\right.$ and $\left.N^{(1)}=6\right)$, and the $j$ th neighboring vector with a neighboring range $k$ is denoted by $n_{j}^{(k)}$.

The intravoxel interaction between calcite and dolomite is given as $I_{0}^{(C, D)}=I_{0}^{(D, C)}=1$ such that the calcite and dolomite are effectively not mixed in the same voxel. To reduce the effect of the experimental noise, small repulsive interactions between the void and the mineral phases are given as $I_{0}^{(O, D)}=I_{0}^{(O, C)}=I_{0}^{(D, O)}=I_{0}^{(C, O)}=0.01$, which is 
similar or smaller than the X-ray CT experimental noise level. For the same reason, a small attractive interaction is given between nearest-neighbor voxels of the same compositional phases $I_{0}^{(O, O)}=I_{0}^{(C, C)}=I_{0}^{(D, D)}=-0.01$. In each voxel, the approximate optimal composition is identified by sub-dividing the composition search interval into 15 divisions for each composition, and the final optimal composition is calculated using a Lagrange multiplier algorithm.

In this region, the average porosity is calculated as $13.9 \%$ and the average volume fractions of the calcite and dolomite are 54.8 and $31.3 \%$, respectively. This is reasonably consistent with the average porosity as measured using mass difference methods of $16.4(4) \%$ at a different region of the sample. The difference could be due to the inhomogeneity of the rock, which will be discussed later. Figure 6 shows distributions of each phase and porosity determined by DCM in a single slice. The complete data set is available at CSIRO Data-Access-Portal (Yang et al., 2014a).

The region of overlap between the DCM reconstructed and Xe K-edge derived porosity distribution has a size of
$790 \times 790 \times 80$ voxels. To improve numerical accuracy, these 80 CT slices have been analyzed using the same DCM software by sub-dividing the composition search interval into 25 divisions for each composition. In the overlap region, the overall porosity is calculated as $17.7 \%$, the overall volume fractions of the calcite and dolomite are 51.3 and $31.0 \%$, respectively. The distributions of the compositions at slice 10 of the overlapping region are shown in Figure 6.

\section{Comparison of Porosity Analysis by Both Methods}

The output of both the DCM and K-edge subtraction analyses include the 3D distribution of the porosity and a measure of the overall porosity enabling a direct comparison of the two methods. It is to be expected that the porosity distribution and percentage porosity (e.g., percentage of void space in the sample volume) determined from K-edge subtraction should be a reasonably accurate reflection of the
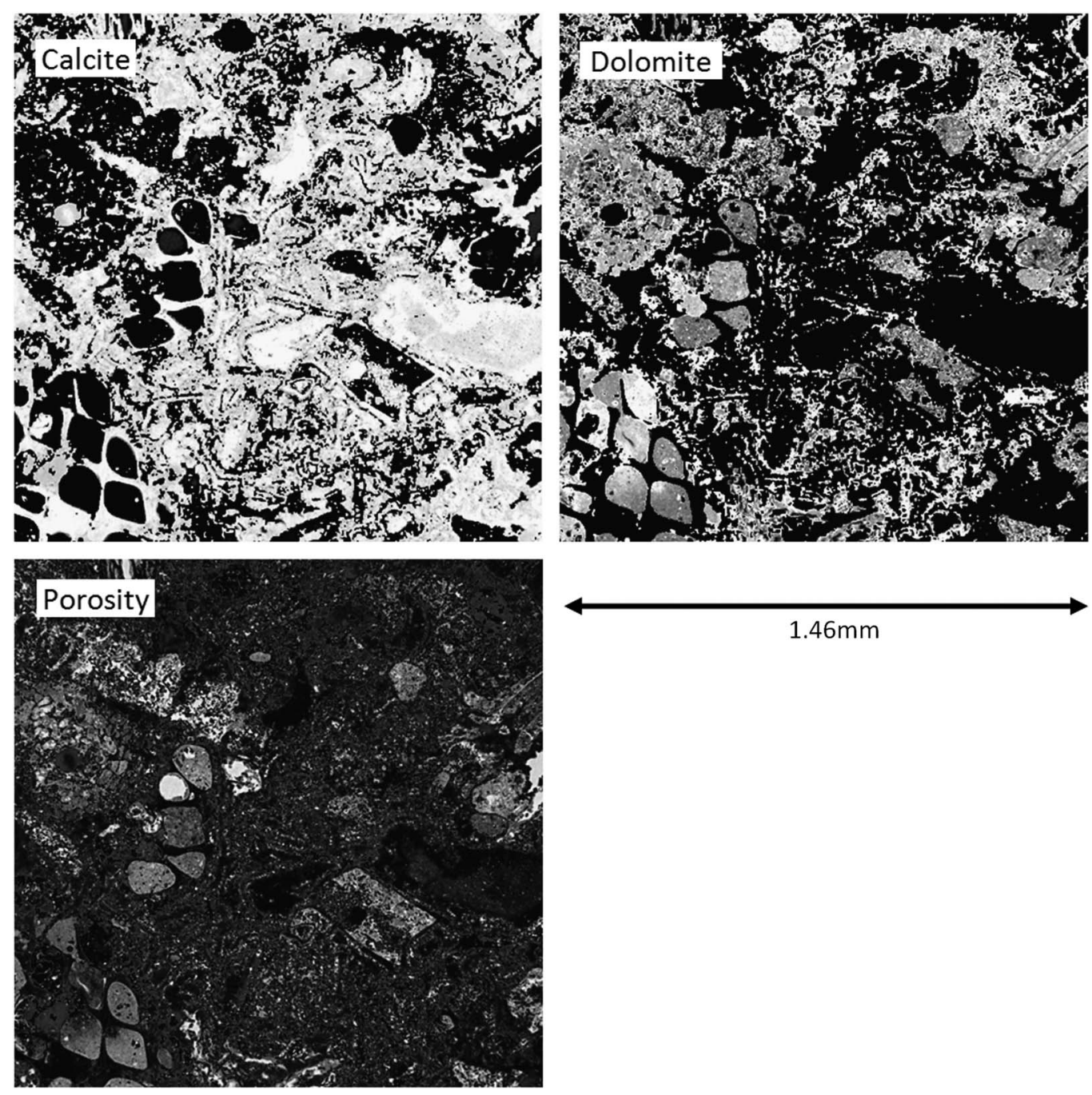

Figure 6. Mineral phase distributions on the 10th slice in the overlap region. Upper left: calcite volume fraction; upper right: dolomite volume fraction; lower left: void fraction. 
open porosity in the sample, within the accuracy of the method, and so can be regarded as providing "ground truth" for validation of porosity modeling using the DCM method in that porous regions detected using xenon should also be present in the DCM model. It should be noted that DCM may also be able to detect closed porous regions, which xenon cannot penetrate.

Overall porosity as determined by DCM for the overlapping region of the two sets of data was $17.5 \%$. For the K-edge subtraction data this value was calculated from the ratio of the average xenon density inside the overlap region of the sample to the xenon density in the pure gas region and was found to be $19.5(4) \%$. It is worth noting that since the xenon can only penetrate connected porous regions, the xenon measured porosity might be expected to be a little smaller than the measurement obtained by DCM; however, the results here show that it is in fact larger. This suggests that some of the porosity is not detected by the DCM modeling method.

Figure 7 shows a comparison between the porosity maps determined using DCM and xenon K-edge subtraction. Since the xenon K-edge map is of lower resolution the DCM image is blurred (Gaussian blur) for ease of visual comparison. In this particular cross section, two regions are shown in which DCM fails to detect porosity seen in the xenon map. In the DCM maps these are often, but not always, identified as pure dolomitic regions. Furthermore, the magnitude of the porosity determined by DCM in high porosity regions is a little lower than that of the xenon map. The ratio of the dolomite to calcite X-ray absorption coefficients changes from $70 \%$ at $28 \mathrm{keV}$ to $73.5 \%$ at $38 \mathrm{keV}$, the two energies used for the DCM map. This small change may be insufficient in some cases for DCM to fully distinguish between void and dolomite content, so that these regions have higher porosity and calcite content than identified by DCM.
The Xe data set was also analyzed for porosity over a larger region than the overlapping segment. By looking at the porosity of small similar sized regions of the sample it was found that porosity varied significantly in different parts of the sample of comparable size to the overlap section, largely driven by the different fossil forms that comprized different regions. Porosity measured over 11 such segments varied between 8 and 23\% with an average value of 14(1)\%, thus the overlap region with the Swiss Light Source data set was one of the more porous parts of the sample. The overall porosity determined by xenon infusion is reasonably consistent with, but a little lower, than the 16.4(1)\% porosity determined for the sample as a whole, using the mass difference method. The difference, however, may just reflect the fact that the xenon measurement only covered part of the sample.

\section{Conclusions}

The comparison of porosity distribution determined by the two methods shows that DCM overall successfully identifies most porous regions, however, some ambiguity between void fraction and the relative fractions of calcite and dolomite may not have been fully resolved in this case. This could be improved by optimizing the X-ray energies during the CT experiments so as to provide maximum absorption contrast between the sample's constituent material phases.

An alternative, though more experimentally demanding, approach would be to combine the DCM with xenon $\mathrm{K}$-edge subtraction. If the sample is immersed in pressurized xenon, micro-CT data sets could be acquired above and below the xenon absorption edge as well as at other energies selected to distinguish the two main mineral phases.
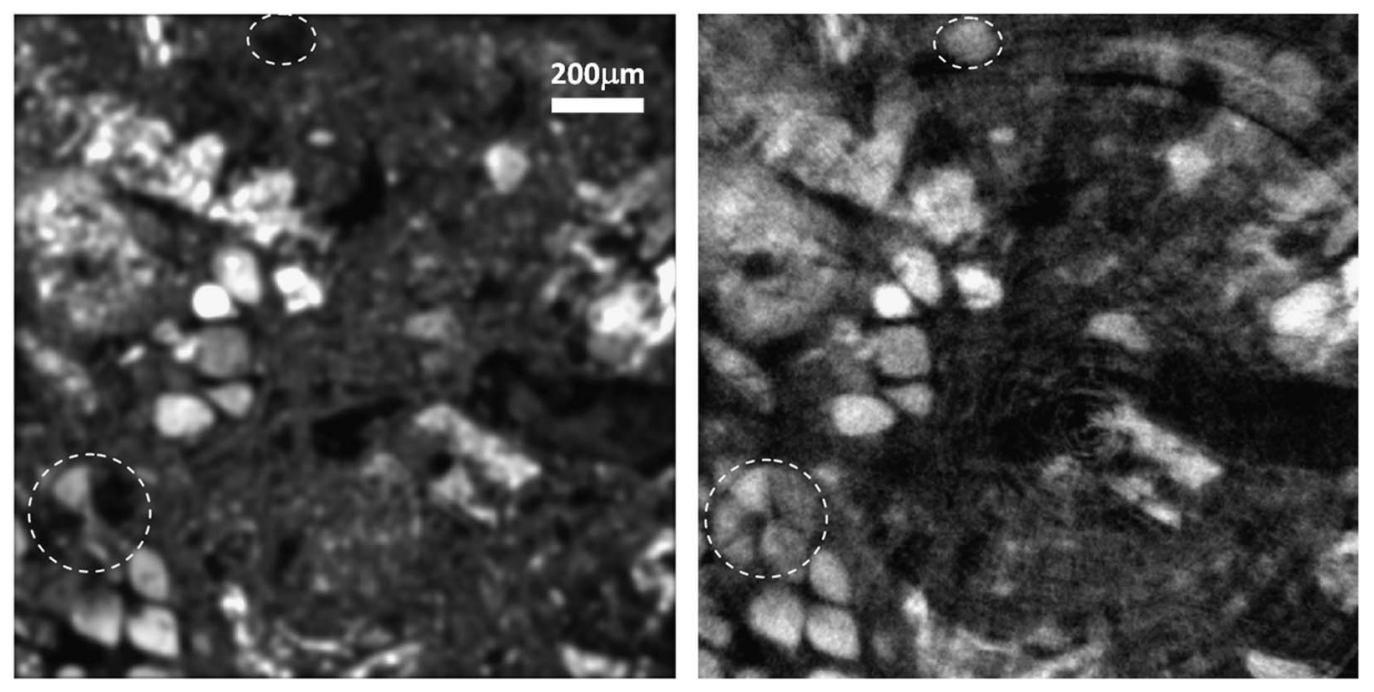

Figure 7. Porosity distribution in the 10th overlap slice determined by DCM porosity distribution blurred (left) for ease of comparison with lower resolution porosity distribution obtained from xenon K-edge subtraction (right). Although the two porosity distributions have good correspondence overall, two regions are highlighted where DCM fails to identify porous regions. The missed pore region by DCM tends to have relatively low X-ray absorption contrast. DCM, data-constrained modeling. 
The K-edge data sets would give very good information on the porosity with high confidence.

One way to use data collected in this way would be to determine the porosity using just the K-edge data and then fixing this value for the DCM analysis. Alternatively, and probably a better approach, would be to include xenon as a phase to be determined along with the mineral phases and void phases. This second method avoids the problems that may occur if there are isolated porous regions in the sample into which the xenon does not penetrate. Close examination of the micro-CT data sets acquired with and without xenon appeared to indicate that there were a few regions with possible small isolated pores, although the majority of the pores were clearly penetrated by the xenon.

\section{ACKNOWLEDGMENTS}

Part of this research was undertaken on the Imaging and Medical beamline at the Australian Synchrotron, Victoria, Australia, and is supported by the Science and Industry Endowment Fund (SIEF). Additional synchrotron experiments were carried out at the TOMCAT beamline at the Swiss Light Source, the authors would like to thank Marco Stampanoni for making beamtime available. The authors would like to thank the MASSIVE team for the great computing facilities they provide.

\section{REFERENCES}

Agbogun, H.M.D., AL, T.A. \& Hussein, E.M.A. (2013). Three dimensional imaging of porosity and tracer concentration distributions in a dolostone sample during diffusion experiments using X-ray micro-CT. J Contam Hydrol 145, 44-53.

Andrew, M., Bijeljic, B. \& Blunt, M.J. (2014). Pore-scale contact angle measurements at reservoir conditions using X-ray microtomography. Adv Water Resour 68, 24-31.

Arns, C.H., Bauget, F., Ghous, A., Sakellariou, A., Senden, T.J., Sheppard, A.P., SoK, R.M., Pinczewski, W.V., Kelly, J.C. \& KNACKSTEDT, M.A. (2005). Digital core laboratory: Petrophysical analysis from 3D imaging of reservoir core fragments. Petrophysics 46(4), 260-277.

Bera, B., Gunda, N.S.K., Mitra, S.K. \& Vick, D. (2012). Characterization of nanometer-scale porosity in reservoir carbonate rock by focused ion beam-scanning electron microscopy. Microsc Microanal 18(1), 171-178.

BerA, B., Mitra, S.K. \& VICK, D. (2011). Understanding the micro structure of Berea sandstone by the simultaneous use of microcomputed tomography (micro-CT) and focused ion beamscanning electron microscopy (FIB-SEM). Micron 42(5), 412-418.

Boone, M.A., Kock, T.D., Bultreys, T., Schutter, G.D., Vontobel, P., Hoorebeke, L.V. \& Cnudde, V. (2014). 3D mapping of water in oolithic limestone at atmospheric and vacuum saturation using X-ray micro-CT differential imaging. Mater Charact 97, 150-160.

Chen, W.H., Yang, S.Y.S., XIAO, T.Q., Mayo, S.C., Wang, Y.D. \& WANG, H.P. (2014). A synchrotron-based local computed tomography combined with data-constrained modelling approach for quantitative analysis of anthracite coal microstructure. J Synch Rad 21(3), 586-593.
Cnudde, V. \& Boone, M. (2013). High-resolution X-ray computed tomography in geosciences: A review of the current technology and applications. Earth Sci Rev 123, 1-17.

Fusi, N. \& Martinez-Martinez, J. (2013). Mercury porosimetry as a tool for improving quality of micro-CT images in low porosity carbonate rocks. Eng Geol 166, 272-282.

Ghous, A., Senden, T., Sok, R., Sheppard, A., Pinczewski, W. \& KNACKSTEDT, M. (2007). Characterisation of microporosity in carbonate cores. In Middle East Regional SPWLA Symposium: Petrophysics and Brown Field Resource Optimization, Society of Petrophysicists and Well Log Analysts (SPWLA), USA (Ed.), pp. 11. Abu Dhabi.

Gureyev, T.E., Nesterets, Y., Ternovski, D., Thompson, D., Wilkins, S.W., Stevenson, A.W., Sakellariou, A. \& Taylor, J.A. (2011). Toolbox for advanced X-ray image processing. $A d v$ Computat Met X-Ray Opt II 8141, 81410B.

Ketcham, R.A. \& IturRinO, G.J. (2005). Nondestructive highresolution visualization and measurement of anisotropic effective porosity in complex lithologies using high-resolution X-ray computed tomography. J Hydrol 302(1-4), 92-106.

Klobes, P., Riesemeier, H., Meyer, K., Goebbels, J. \& Hellmuth, K.H. (1997). Rock porosity determination by combination of X-ray computerized tomography with mercury porosimetry. Fresenius J Anal Chem 357(5), 543-547.

Mayo, S.C., Tulloh, A.M., Trinchi, A. \& YAng, S.Y.S. (2012). Dataconstrained microstructure characterization with multispectrum X-ray micro-CT. Microsc Microanal 18(3), 524-530.

Nugent, K., Gureyev, T., Cookson, D., Paganin, D. \& Barnea, Z. (1996). Quantitative phase imaging using hard X rays. Phys Rev Lett 77(14), 2961.

Paganin, D., Mayo, S.C., Gureyev, T.E., Miller, P.R. \& Wilkins, S.W. (2002). Simultaneous phase and amplitude extraction from a single defocused image of a homogeneous object. J Microsc 206, 33-40.

Pinczewski, W.V., Kelly, J.C. \& Knackstedt, M.A. (2005). Digital core laboratory: Petrophysical analysis from 3D imaging of reservoir core fragments. Petrophysics 46(4), 260-277.

Snigirev, A., Snigireva, I., Kohn, V., KuZnetsov, S. \& Schelokov, I. (1995). On the possibilities of X-ray phase contrast microimaging by coherent high-energy synchrotron radiation. Rev Sci Instrum 66(12), 5486-5492.

Trinchi, A., Yang, Y.S., Huang, J.Z., Falcaro, P., Buso, D. \& CAO, L.Q. (2012). Study of 3D composition in a nanoscale sample using data-constrained modelling and multi-energy X-ray CT. Model Simul Mater Sci Eng 20(1), 015013.

Umetani, K., Ueda, K., Takeda, T., Akisada, M., Nakajima, T. \& Anno, I. (1991). Iodine K-edge dual-energy imaging for subtraction angiography using synchrotron radiation and a 2-dimensional detector. Nucl Instrum Met Phys Res Sec A 301(3), 579-588.

Van Geet, M., Swennen, R. \& Wevers, M. (2000). Quantitative analysis of reservoir rocks by microfocus X-ray computerised tomography. Sediment Geol 132(1-2), 25-36.

WANG, H.P., YANG, Y.S., WANG, Y.D., YANG, J.L., JiA, J. \& NiE, Y.H. (2013a). Data-constrained modelling of an anthracite coal physical structure with multi-spectrum synchrotron X-ray CT. Fuel 106, 219-225.

Wang, Y.D., YANG, Y.S., Cole, I., Trinchi, A. \& XIAO, T.Q. (2013b). Investigation of the microstructure of an aqueously corroded zinc wire by data-constrained modelling with multi-energy X-ray CT. Mater Corros-Werkstoffe Und Korrosion 64(3), 180-184. 
Wang, Y.D., Yang, Y.S., XIAO, T.Q., Liu, K.Y., Clennell, B., Zhang, G.Q. \& WANG, H.P. (2013c). Synchrotron-based data-constrained modeling analysis of microscopic mineral distributions in limestone. Int J Geosci 4, 344-351.

Wilkins, S.W., Gureyev, T.E., Gao, D., Pogany, A. \& Stevenson, A.W. (1996). Phase-contrast imaging using polychromatic hard X-rays. Nature 384(6607), 335-338.

WithJACK, E.M. \& AKervoll, I. (1988). Computed tomography studies of 3-D miscible displacement behavior in a laboratory five-spot model. In SPE Annual Technical Conference and Exhibition, SPE-18096-MS. Houston, TX: Society of Petroleum Engineers.

Yang, S., Mayo, S., Pervukhina, M., Clennell, B., Esteban, L., Irvine, S., Siu, K., Maksimenko, A. \& Tulloh, A. (2014a). Carbonate rock X-ray CT and DCM microstructure data. v1. CSIRO. Data Collection. 10.4225/08/5476787E08766.

Yang, S., Tulloh, A., Chu, C., Chen, F., \& TAylor, J. (2014b). DCM A software platform for advanced 3D materials modelling, characterisation and visualization. v9. CSIRO. Data Collection. 10.4225/08/55371BD329075.
Yang, Y., Tulloh, A., Muster, T., Trinchi, A., Mayo, S. \& Wilkins, S. (2010a). Data-constrained microstructure modeling with multi-spectrum X-ray CT. In SPIE 7804, Developments in X-Ray Tomography VII, 78040N, doi: $10.1117 / 12.861964$.

Yang, Y.S., Gureyev, T.E., Tulloh, A., Clennell, B. \& Pervukhina, M. (2010b). Feasibility of a data-constrained prediction of hydrocarbon reservoir sandstone microstructures. Meas Sci Technol 21, 047001.

Yang, Y.S., Liu, K.Y., MaYo, S., Tulloh, A., Clennell, M.B. \& XIAO, T.Q. (2013a). A data-constrained modelling approach to sandstone microstructure characterisation. J Petrol Sci Eng 105, 76-83.

Yang, Y.S., Tulloh, A., Chen, F., Liu, K.Y., Clennell, B. \& Taylor, J. (2013b). Data-constrained characterization of sandstone microstructures with multi-energy X-ray CT. 11th International Conference on X-Ray Microscopy (XRM2012). J Phys: Conf Ser. 463, 012048. 